\title{
A Reconfigurable Hybrid Wheel-track Mobile Robot Based on Watt II Six-bar Linkage
}

\author{
Zirong Luo ${ }^{\mathrm{a}, *}$, Jianzhong Shang ${ }^{\mathrm{a}}$, Guowu Wei ${ }^{\mathrm{b}, *}$, Lei Ren ${ }^{\mathrm{c}, *}$ \\ ${ }^{a}$ College of Mechatronic Engineering and Automation, National University of Defense Technology, Changsha 410073, P.R. China \\ ${ }^{b}$ School of Computing, Science and Engineering, University of Salford, Salford M5 4WT, United Kingdom \\ ${ }^{c}$ School of Mechanical, Aerospace and Civil Engineering, University of Manchester, Manchester M13 9PL, United Kingdom
}

\begin{abstract}
This paper presents the design and development of a novel reconfigurable hybrid wheel-track mobile robot (RHMBot). This new reconfigurable mobile robot is constructed based on a Watt II six-bar linkage; through structure reconfiguration, it can provide three locomotion modes as wheel mode, tracked mode, and climbing and roll-over mode. Mechanical design of the proposed RHMBot is introduced, and using mechanism decomposition kinematics of the reconfigurable frame is investigated. Locomotion of the robot is then interpreted associated with transformation of the reconfigurable frame. Further, deformation of the deformable track belt is characterized and static analysis of the reconfigurable frame is accomplished. Numerical simulation of the proposed reconfigurable frame is subsequently implemented, integrated with driving-torque associated parametric study, leading to optimization of the structure parameters. Consequently, prototype of the proposed RHMBot is designed and developed; exploiting which a series of field tests are conducted verifying feasibility and manoeuvrability of the proposed multilocomotion mobile robot.
\end{abstract}

Keywords: Hybrid wheel-track mobile robot, Watt II six-bar linkage, reconfigurable robot, kinematics and statics, parametric study

\section{Introduction}

Mobile robots have been widely used in civilian tasks and military missions, such as field and space exploration, disaster rescue, reconnaissance and surveillance [1]. They are normally demanded to enter into unstructured, severe and hostile environments equipped with various devices. In these scenarios, mobility and manoeuvrability in rugged terrain is a critical criterion for mobile robot design.

Bruzzone and Quaglia [2] reviewed the locomotion systems of ground mobile robots which can be classified into wheeled robots [3], legged robots [4], tracked robots [5], wheel-legged robots [6], wheeltracked robots [7], wheel-legged-tracked robots [8] and hybrid mobile robot [9]. Usually, the tracked mobile robots have excellent terrain adaptability, full mobility and obstacle overcoming capability. Hence, they are widely used in military and security areas and their locomotion mechanisms are usually of different forms, such as 2-parallel-track [10], leg-track [11], 4-track [12, 13, 14] and 6-track [15] etc.

Compared with the conventional robots, the reconfigurable robots [16, 17] can alter their structures and configuration to better adapt to various, complicated and completely unstructured environment. Considering the concept of reconfigurability, to overcome the weakness of tracked robots with two par15 allel tracks, the IRobot ${ }^{\circledR}$ developed the PackBot ${ }^{\mathrm{TM}}$ series with variable track structures [18]; according to the planned tasks, the robots can be equipped with a pair of forward tracks or pairs of forward and backward tracks, rotation of the tracks are intended to assist the robots to overcome obstacles. Li et al. [19] developed a transformable wheel-track robot, which can change its track configuration and locomotion mode. Such a robot works like a military tank when the track is in lower positions and changes its track

\footnotetext{
${ }^{*}$ Corresponding author

Email addresses: luozirong@nudt. edu.cn (Zirong Luo ), g. wei@salford.ac . uk (Guowu Wei ), lei.ren@manchester.ac.uk (Lei Ren)
} 
into triangular shape so as to improve its capability of adaptation to complex terrain with obstacles [20]. Kim [21] et.al proposed a wheel-and-track integrated mobile platform with terrain adaptive capability. Ben-Tzvi, et.al [9] presented an articulated hybrid mobile robot that has the capability of locomotion as well as manipulation. In 2009, the Elbit ${ }^{\circledR}$ company [22] 23] developed a reconfigurable robot VIPeR ${ }^{\mathrm{TM}}$, which integrated the virtues of wheeled and tracked robots. As the track can be contracted into the wheel by a track transformational mechanism, the robot has two kinds of locomotion configurations: wheeledand tracked-configuration.

Inspired by the aforementioned VIPeR ${ }^{\mathrm{TM}}$ mobile robot, a novel reconfigurable hybrid wheel-track mobile robot (RHMBot) [24, with multiple locomotion modes is presented in this paper, as shown in Fig. 1] Comparing with the VIPeR mobile robot [23] whose reconfigurable frame is constructed by two branches of six-bar linkage driven by two linear hydraulic actuators; the reconfigurable frame of the proposed RHMBot is constructed by two branches four-bar linkage forming a united Watt II six-bar linkage [25] actuated by only one rotary electronic motor. The structure and control for the proposed RHMBot are much simpler than the VIPeR mobile robot. Although six-bar linkages have been widely used in various engineering applications [26, 27], it is the first time that it is used in the development of a reconfigurable

35 hybrid wheel-track mobile robot. The proposed RHMBot can perform three locomotion modes, while VIPeR only has two mode as wheel mode and tracked mode; a new mode of climbing and rolling (not available for VIPeR) enables the RHMBot to overcome higher obstacles.

Mechanical design fo the RHMBot is introduced and kinematics of the Watt II six-bar linkage is presented. Locomotions of the robot with respect to the six-bar linkage is revealed and static analysis associated with deformation of the deformable track belt is carried out. Position synchronization is then accomplished and prototype of the proposed RHMBot is developed leading to the field tests verifying the feasibility and manoeuvrability.

\section{Mechanical Design of a Reconfigurable Hybrid Wheel-track Mobile Robot}

The reconfigurable hybrid wheel-track mobile robot (RHMBot) presented in this paper is a modular multi-locomotion robot. As illustrated in Fig. 17a, it consists of a chassis for accommodating the rollover mechanism, transmission system and control system; a pair of reconfigurable frames; two pairs of geared wheels; a pair of deformable track belts wrapping the geared wheels and reconfigurable frames for adapting locomotions; and a tail frame. The robot works in the wheeled mode when it moves on an even and smooth ground to obtain better efficiency, as shown in Fig. 1 $1 \mathrm{~b}$; it transforms into the tracked $1 \mathrm{k}$; in the case that it encounters obstacles such as vertical stairs, the robot will transform itself into the climbing mode with the assistance from the tail frame, in this case, the tracked wheel will rotate as a wheel to overcome higher obstacle, as illustrated in Fig. $1 \mathrm{~d}$.

Reconfigurability of the robot's locomotion modes is implemented by a novel reconfigurable system, which comprises a actuating system, and a pair of symmetric reconfigurable frames that are embedded in two geared track wheels and each of which is made of a Watt II six-bar linkage. As shown in Fig. 2a, the actuating system contains the motor, the worm gear, the main shaft, the driving frame and the hollow shaft, which provides driving torque for the reconfigurable frame. The reconfigurable frame is mainly made up of a Watt II linkage which contains the V-shaped link, T-shaped link, link A, link B, link E and link

6o F; and together with the geared track wheels leading to reconfiguring the deformable track belt from the wheeled shape to the triangular-tracked shape or vice versa.

This design also benefits from the use and construction of a deformable track belt which is made of three independent layers, as illustrated in Fig. 2b, i.e., an elastic layer, a transmission layer and a constraint layer. The elastic layer is an internal layer, which is used to provide initial tension for maintaining the mesh between the geared wheel and transmission layer; this layer can adjust itself to fit the length of the constraint layer. The transmission layer is an intermediate layer consisting of ten separated gearshape teeth that mesh with the geared wheels. The constraint layer is an external layer with constant length, it is exploited to limit the maximum length of the deformable track belt and to ensure the correct track transmission without being influenced by the deformation of the elastic layer. Further, since the constraint layer is flexible, it can change its shape according to the deformation of the track to fit various configurations. 


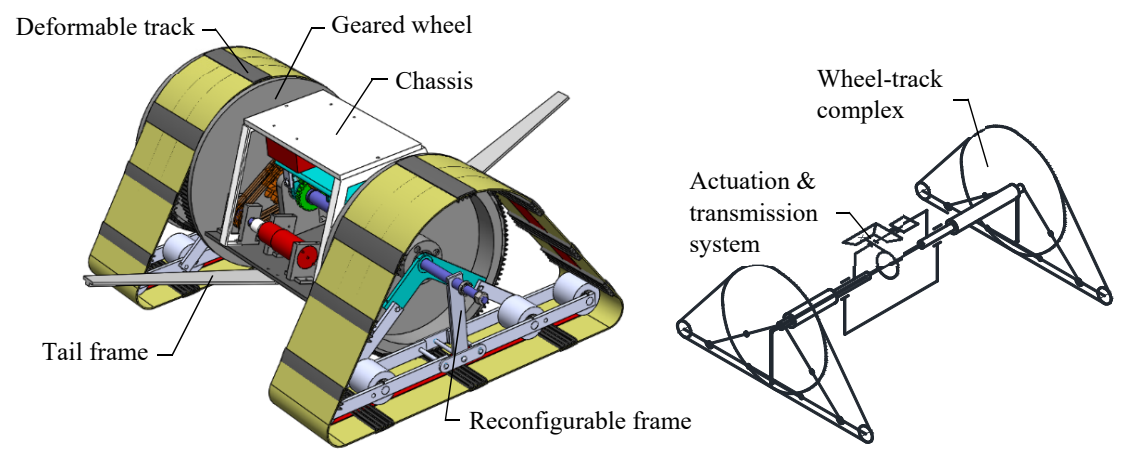

(a) Mechanical structure and schematic diagram of the RHMBot

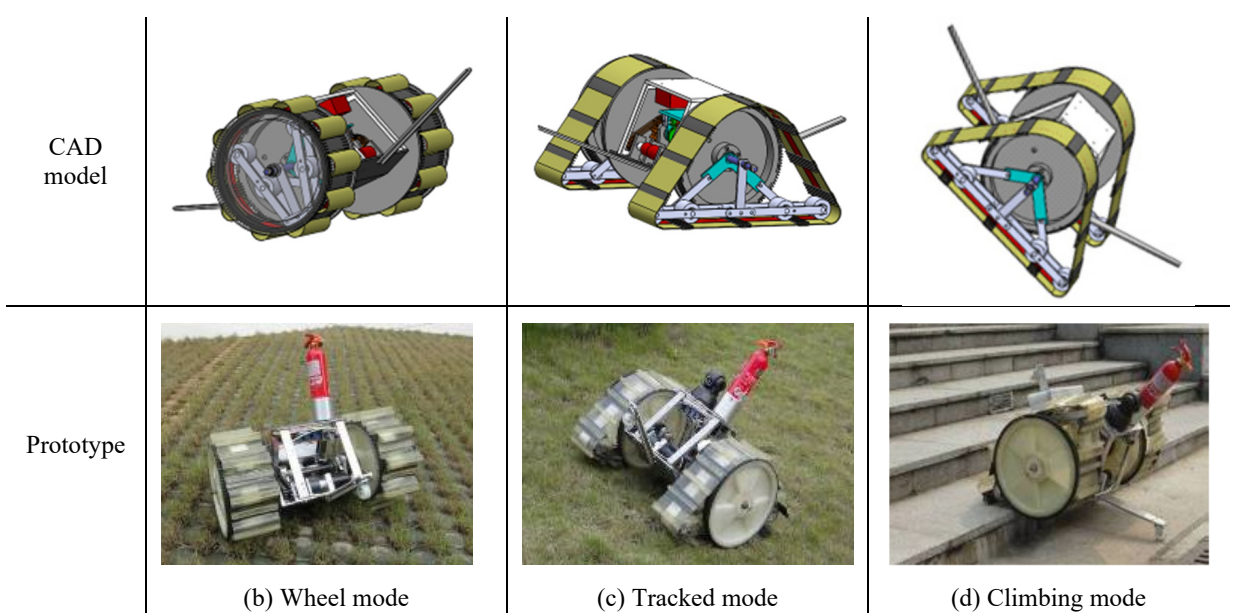

Figure 1: Mechanical structure of the RHMBot and the three modes

As shown in Fig. 2k, when the whole reconfigurable frame retracts into the internal space of the track wheel, the robot works in the wheeled mode; in this mode, the deformable track belt remains meshed with the track wheel and roll together with the track wheel. In the cases that the robot works on irregular and rough terrain or encounters obstacles, the reconfigurable frame will be expanded leading to the tracked locomotion mode by altering the shape of the deformable track belt, see Fig. $2 \mathrm{~d}$ and $2 \mathrm{e}$. Hence, by reconfiguring of the locomotion reconfigurable system, the robot can work in three modes, i.e., wheeled mode, tracked mode and climbing mode; with the shape of the deformable track belt adapted so as to enveloping the supporting wheels and the geared wheel as illustrated in Figs. 20: - h.

\section{Kinematics of the Watt II Six-bar Linkage and Configurations of the Mobile Robot}

Based on the aforementioned mechanical design, it can be found that transformation of the locomotions of the robot is achieved through reconfiguration of the reconfigurable frame which is constructed using a Watt II six-bar linkage. In order to reveal the relation between configurations of the reconfigurable frame and locomotions of the robot, in this section kinematics of the linkage is investigated and configuration of the hybrid wheel-track structure is characterized.

\subsection{Kinematics of the Watt II Six-bar Linkage and Positions of the Two Extension Wheels}

Corresponding to the wheel-track transformation system indicated in Fig.2 . without loss of generality, schematic diagram of the Watt II six-bar linkage, in the reconfigurable frame associated with the track wheel and the deformable track belt, is presented in Fig. 3 Where, link ADH is a ternary T-shaped link, and link $\mathrm{ABF}$ is a ternary V-shaped link; the rest of the links are binary elements. By taking the T-shaped link as a frame which is mounted at the centre A of the track wheel, a Cartesian coordinate system is 


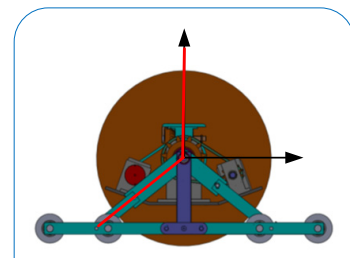

(c) tracked mode

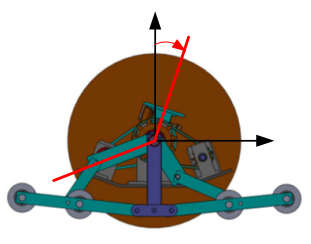

(d) expansion mode

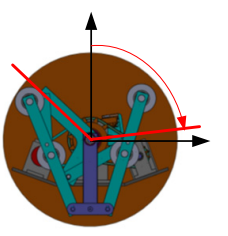

(e) wheeled mode

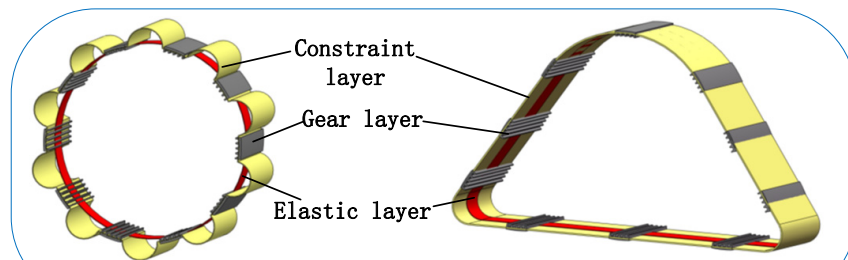

(b) Structure of the deformable track belt

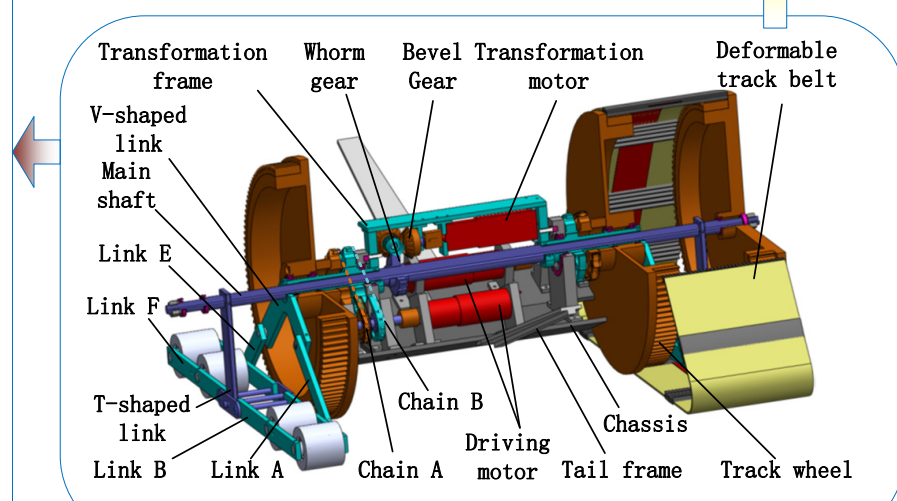

(a) the reconfiguratable system

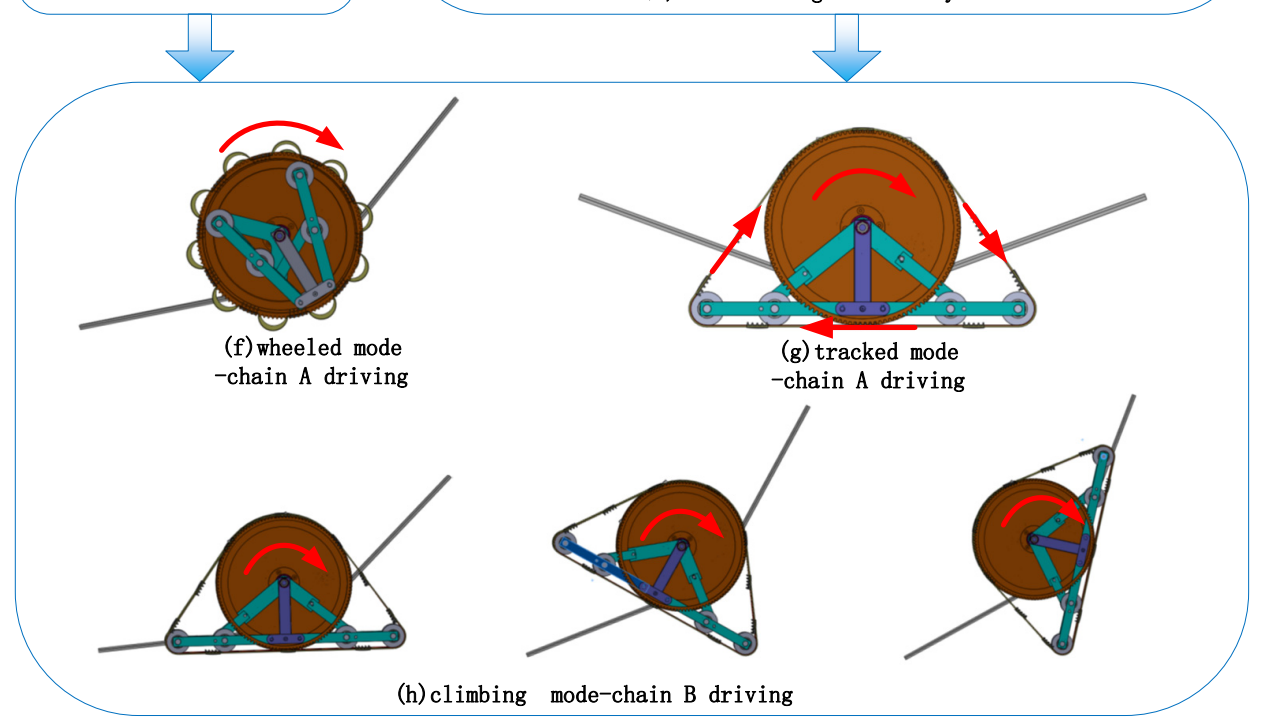

Figure 2: Detailed design of the RHMBot and modes of the reconfigurable frame 


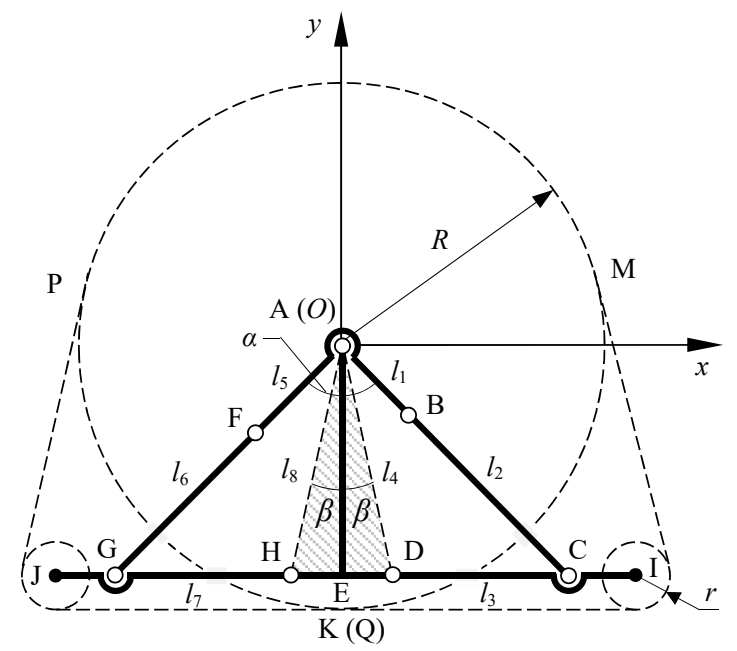

Figure 3: Geometry of the Watt II Six-bar linkage in the reconfigurable frame

established with its origin located at point A, $y$-axis aligns with AE which is perpendicular bisector of segment DH and $x$-axis being positive in the rightward direction. Further, the geometric parameters are defined as: 1) Point A is the centre of the track wheel whose radius is $R$, points I and J are centres of the two track belt extension wheels with $r$ denoting their radius; 2) $l_{1}, l_{2}, l_{3}$ and $l_{4}$ denote the lengths of links AB, $\mathrm{BC}, \mathrm{CD}$ and $\mathrm{AD}$ of the sub-branch four-bar linkage $\mathrm{ABCD}$, and $l_{5}, l_{6}, l_{7}$ and $l_{8}$ denote the lengths of links AF, FG, GH and AH of the other sub-branch four-bar linkage AFGH; the length of segments $\mathrm{CI}$ and GJ are denoted as $l ; 3$ ) The angle between $\mathrm{AB}$ and $\mathrm{AF}$ is denoted as $\alpha$ and the angles between $\mathrm{AE}$ and $\mathrm{AD}$, and $\mathrm{AE}$ and $\mathrm{AH}$ are both $\beta ; 4)$ The joint angles $\theta_{1}$ throughout $\theta_{8}$ are defined as the angles between the $x$-axis and link $l_{i}(i=1,2, \ldots, 8)$ starting from positive direction of the $x$-axis and ending at link $l_{i}$ counter-clockwise. In addition, referring to Fig. 3 there exists an intrinsic geometric constraint for the structure parameters as $\beta=\arccos \left((R-r) / l_{4}\right)=\arccos \left((R-r) / l_{8}\right)$.

During the locomotion transformation process, the total length of the deformable track belt is mainly determined by positions of the two extension wheels attached at points I and J, hence, in this section, displacements of the extension wheels at I and J are investigated based on the geometry of the Watt II six-bar linkage. Using mechanism decomposition, the Watt II six-bar linkage can be treated as two subbranches of four-bar linkages, i.e. four-bar linkages ABCD and AFGH. Displacement of extension wheel I can be obtained in linkage ABCD and displacement of wheel $\mathrm{J}$ can be deduced in linkage AFGH.

In four-bar linkage ABCD based on loop equation 28 it has

$$
\left[\begin{array}{cc}
\cos \theta_{1} & \cos \theta_{2} \\
\sin \theta_{1} & \sin \theta_{2}
\end{array}\right]\left[\begin{array}{l}
l_{1} \\
l_{2}
\end{array}\right]=\left[\begin{array}{cc}
\cos \theta_{3} & \cos \theta_{4} \\
\sin \theta_{3} & \sin \theta_{4}
\end{array}\right]\left[\begin{array}{l}
l_{3} \\
l_{4}
\end{array}\right]
$$

where $\theta_{1}$ is the input angle and referring to Fig. 3 it has angle $\theta_{4}=3 \pi / 2+\beta$.

Solving Eq. (1) leads to the two variables, i.e. angles $\theta_{2}$ and $\theta_{3}$ as,

$$
\theta_{3}=\arctan \left(\frac{V}{U}\right) \pm \arccos \left(\frac{W}{\sqrt{U^{2}+V^{2}}}\right)
$$

with $U=2 l_{3}\left(l_{4} \cos \theta_{4}-l_{1} \cos \theta_{1}\right), V=2 l_{3}\left(l_{4} \sin \theta_{4}-l_{1} \sin \theta_{1}\right)$ and $W=l_{2}{ }^{2}+2 l_{1} l_{4} \cos \left(\theta_{1}-\theta_{4}\right)-l_{1}{ }^{2}-l_{3}{ }^{2}-l_{4}{ }^{2}$; and

$$
\theta_{2}=\arctan \left(\frac{V_{1}}{U_{1}}\right) \pm \arccos \left(\frac{W_{1}}{\sqrt{U_{1}^{2}+V_{1}^{2}}}\right)
$$

with $U_{1}=2 l_{2}\left(l_{1} \cos \theta_{1}-l_{4} \cos \theta_{4}\right), V_{1}=2 l_{2}\left(l_{1} \sin \theta_{1}-l_{4} \sin \theta_{4}\right)$ and $W_{1}=l_{3}{ }^{2}+2 l_{1} l_{4} \cos \left(\theta_{1}-\theta_{4}\right)-l_{1}{ }^{2}-l_{2}{ }^{2}-l_{4}{ }^{2}$. 
Thus, position of point I can be presented as

$$
\boldsymbol{p}_{I}=\left[\begin{array}{c}
x_{I} \\
y_{I}
\end{array}\right]=\left[\begin{array}{cc}
\cos \theta_{4} & \cos \theta_{3} \\
\sin \theta_{4} & \sin \theta_{3}
\end{array}\right]\left[\begin{array}{c}
l_{4} \\
l_{3}+l
\end{array}\right]
$$

which can also be obtained as $\boldsymbol{p}_{I}=\boldsymbol{l}_{1}+\boldsymbol{l}_{2}+\boldsymbol{l}_{C I}$; where $\boldsymbol{l}_{1}$ and $\boldsymbol{l}_{2}$ are vectors for links AB and BC, and $\boldsymbol{l}_{C I}$ is a vector from point $\mathrm{C}$ to point $\mathrm{I}$.

Similarly, in the sub-branch four-bar linkage AFGH, position of point J can be deduced as

$$
\boldsymbol{p}_{J}=\left[\begin{array}{c}
x_{J} \\
y_{J}
\end{array}\right]=\left[\begin{array}{cc}
\cos \theta_{8} & \cos \theta_{7} \\
\sin \theta_{8} & \sin \theta_{7}
\end{array}\right]\left[\begin{array}{c}
l_{8} \\
l_{7}+l
\end{array}\right]
$$

where $\theta_{8}=3 \pi / 2-\beta$, and using loop equation it has

$$
\theta_{7}=\arctan \left(\frac{M}{L}\right) \pm \arccos \left(\frac{N}{\sqrt{L^{2}+M^{2}}}\right)
$$

in which $L=2 l_{7}\left(l_{8} \cos \theta_{8}-l_{5} \cos \theta_{5}\right), M=2 l_{7}\left(l_{8} \sin \theta_{8}-l_{5} \sin \theta_{5}\right)$ and $N=l_{6}{ }^{2}+2 l_{5} l_{8} \cos \left(\theta_{5}-\theta_{8}\right)-l_{5}^{2}-$ $l_{7}^{2}-l_{8}^{2}$. The angle $\theta_{5}$ is the input angle associated with $\theta_{1}$ as $\theta_{5}=\theta_{1}-\alpha$.

In addition, angle $\theta_{6}$ can be obtained as

$$
\theta_{6}=\arctan \left(\frac{M_{1}}{L_{1}}\right) \pm \arccos \left(\frac{N_{1}}{\sqrt{L_{1}^{2}+M_{1}^{2}}}\right)
$$

where $L_{1}=2 l_{6}\left(l_{5} \cos \theta_{5}-l_{8} \cos \theta_{8}\right), M_{1}=2 l_{6}\left(l_{5} \sin \theta_{5}-l_{8} \sin \theta_{8}\right)$ and $N_{1}=l_{7}^{2}+2 l_{5} l_{8} \cos \left(\theta_{5}-\theta_{8}\right)-l_{5}^{2}-$ $l_{6}^{2}-l_{8}^{2}$.

Further, in order to ensure that the reconfigurable frame can be fully contracted and expanded, the structure parameters of the Watt II six-bar linkage must satisfy the constraints that $\left(l_{1}+l_{2}\right) \cos (\alpha / 2)=$ $\left(l_{5}+l_{6}\right) \cos (\alpha / 2)=l_{4} \cos \beta=l_{8} \cos \beta=R-r$ and $l_{3}=l_{7}=(R-r)(\tan (\alpha / 2)-\tan \beta)$, where, $R$ is the radius of the track wheel and $r$ is the radius of the extension wheel.

In addition, in order to realise parametric design of the proposed mobile robot, six design variables are defined which are used to determine the structure parameters, i.e. link lengths of the reconfigurable frame. The six design variables are $\left(R, r, \alpha, \beta, w, p_{1}, p_{5}\right)$, where $R$ is radius of the track wheel, $r$ is radius of the extension wheel, $\alpha$ is the angle between $\mathrm{AB}$ and $\mathrm{AF}$, and $\beta$ is the angle between $\mathrm{AE}$ and AD (AH), as illustrated in Fig. 3 the other three design variables are defined as

$$
\left\{\begin{array}{l}
w=l_{I J_{\max }} \\
p_{1}=l_{1} /\left(l_{1}+l_{2}\right) \\
p_{5}=l_{5} /\left(l_{5}+l_{6}\right)
\end{array}\right.
$$

where $w=l_{I J_{\max }}$ is the distance between the centre points of the two extension wheels I and J when the reconfigurable frame is fully expended.

Using these six design variables, structure parameters, i.e., link lengths of the Watt II six-bar can be determine from the configuration indicated in Fig. 3 as

$$
\left[\begin{array}{llll}
l_{1} & l_{2} & l_{3} & l_{4} \\
l_{5} & l_{6} & l_{7} & l_{8}
\end{array}\right]=(R-r)\left[\begin{array}{llll}
\frac{p_{1}}{\cos (\alpha / 2)} & \frac{1-p_{1}}{\cos (\alpha / 2)} & \frac{\tan (\alpha / 2)}{2}-\tan \beta & \frac{1}{\cos \beta} \\
\frac{p_{5}}{\cos (\alpha / 2)} & \frac{1-p_{5}}{\cos (\alpha / 2)} & \frac{\tan (\alpha / 2)}{2}-\tan \beta & \frac{1}{\cos \beta}
\end{array}\right]
$$

and

$$
l=\frac{w}{2}-(R-r) \tan (\alpha / 2)
$$

where $l$ is the length between the centre point $\mathrm{I}(\mathrm{J})$ and point $\mathrm{C}(\mathrm{G})$ as shown in Fig. 3 . 


\subsection{Configurations and Locomotions of the Mobile Robot}

With the reconfiguration of the Watt II six-bar linkage, the two extension wheels can not only be fully folded embedding inside the track wheel (see Fig. 22) forming wheeled mode locomotion but also be fully expended forming tacked mode locomotion (see Fig. 22). Through reconfiguration of locomotion the robot can work in three modes, i.e. wheeled mode, tracked mode and climbing mode as indicated in Fig. 4 The wheeled mode is mainly suitable for running on even terrain at high speed. In this mode, while the robot encounters a small obstacle, the tail frame (see Fig. 11a) can provide conversion torque for supporting the chassis and help conquer the obstacle as shown in Fig. 4 a.

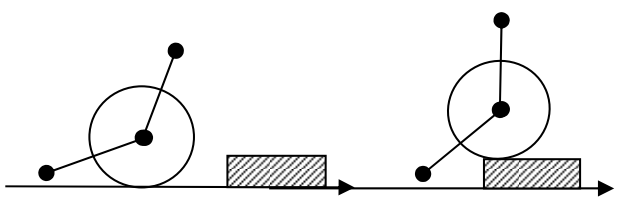

(a) Wheeled mode

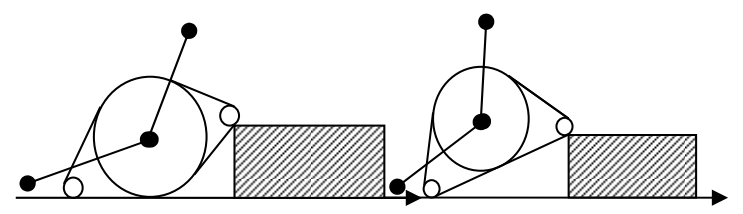

(b) Tracked mode

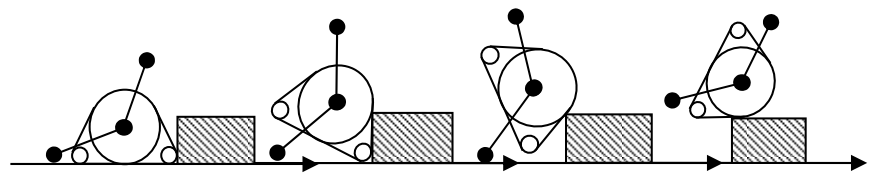

(c) Climbing and rollover mode

Figure 4: Locomotion modes of the mobile robot

When the robot comes across big obstacles and complicated terrains, by reconfiguring the reconfigurable frame, the circular track wheel can be transformed into a triangular track (see Figs. 1 c and d), and correspondingly the wheeled robot is transformed into a tracked robot. As shown in Fig. $4 \mathrm{~b}$ under the tracked mode the robot has better obstacle overcoming capability and can run over sands, grass and gravel path.

In the case that the robot encounters a even larger obstacle that cannot be conquered by the two aforementioned locomotions, the robot will drive the whole triangular track by keeping the triangular track unchanged and rotate the whole body as a wheel to overcome the obstacle with the opposite torque provided by the tail frame. The climbing and roll-over mode is illustrated in Fig. 44.

\section{Deformation of the Deformable Track Belt and Static Analysis}

When the reconfigurable frame changes its configuration, the deformable track belt correspondingly adapts itself to enveloping the track wheel and the extension wheels I and J. In this process, the elastic layer of the deformable track belt extends but the transmission layer and constraint layer keep the same length. In this section, deformation of the deformable track belt is characterized and the static analysis of the two extension wheels related to the Watt II six-bar linkage is analysed. It should be noted that for simplicity purpose, when calculating the length variation of the elastic layer during the transformation process, it is assumed that thickness of the deformable track belt is negligible in the calculation.

\subsection{Geometric Modelling of the Deformable Track Belt}

The deformable track belt has a small deformation to prevent the track from slipping off when the robot works in wheeled mode, deformation occurs in the tracked mode in which case the two extension wheels are stretched outside the track wheel as shown in Fig. 5. Deformation of the deformable track 
belt can be analysed geometrically; without loss of generality, we consider the configuration shown in Fig. 5. which indicates that the deformable track belt changes its length to envelop the track wheel and the two extension wheels. In the figure, MN and KL are two external tangent lines between the track wheel and wheel I, and PU and QV are two external tangent lines between the track wheel and wheel J. In this configuration, positions of points I and J are determined by Eqs. (4) and (5). Let $L_{A I}$ and $L_{A J}$ denote the lengths of vectors $\boldsymbol{p}_{I}$ and $\boldsymbol{p}_{J}$, it has $L_{A I}=\sqrt{x_{I}^{2}+y_{I}^{2}}$ and $L_{A J}=\sqrt{x_{J}^{2}+y_{J}^{2}}$, where $x_{I}, y_{I}, x_{J}$ and $y_{J}$ can be found in Eqs. (4) and (5). Thus, referring to Fig. 5lengths of the external tangent lines are $L_{M N}=L_{K L}=\sqrt{L_{A I}^{2}-(R-r)^{2}}$ and $L_{P U}=L_{Q V}=\sqrt{L_{A J}^{2}-(R-r)^{2}}$, which leads to the results that

$$
\gamma_{1}=\cos ^{-1}\left(\frac{R-r}{L_{A I}}\right) \text { and } \gamma_{2}=\cos ^{-1}\left(\frac{R-r}{L_{A J}}\right)
$$

where angles $\gamma_{1}$ and $\gamma_{2}$ are illustrated in Fig. 5 .

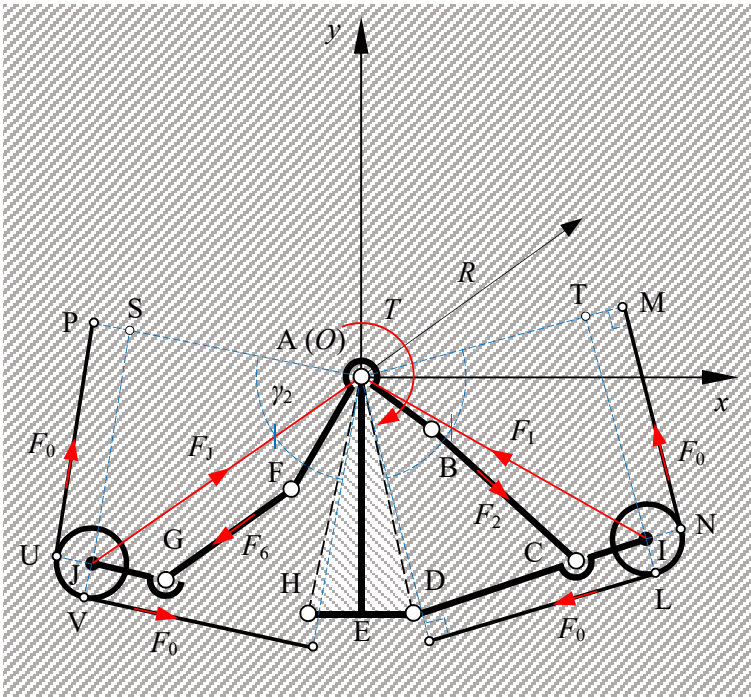

Figure 5: Deformation and static forces of the deformable track belt

When the robot works in the wheeled mode, without any deformation the total length of the deformable track belt is $2 R \pi$, which is equal to the circumference of the track wheel. When the robot works in the tracked mode, the deformable track belt is extended and the deformation of the length can be expressed as

$$
\begin{aligned}
\Delta_{L} & =L_{M N}+2 r \gamma_{1}+L_{K L}+L_{P U}+2 r \gamma_{2}+L_{Q V}-2 R\left(\gamma_{1}+\gamma_{2}\right) \\
& =2\left(\sqrt{L_{A I}^{2}-(R-r)^{2}}+\sqrt{L_{A J}^{2}-(R-r)^{2}}+(r-R)\left(\gamma_{1}+\gamma_{2}\right)\right)
\end{aligned}
$$

In the case that either $R-r>L_{A I}$ or $R-r>L_{A J}, \gamma_{1}$ or $\gamma_{2}$ in Eq. 11] becomes unnecessary, which physically implies that supporting wheels I or J are embedded inside the track wheel.

Hence, by defining the original length of the deformable track belt as $L_{0}=2 R \pi$ and considering the deformation in Eq. 12, length of the deformable track belt in general configuration can be expressed as

$$
L_{T}=L_{0}+\Delta_{L}
$$

and the ratio between the deformation (elongation) and the original length, i.e. strain is defined as

$$
\varepsilon=\frac{\Delta_{L}}{L_{0}}
$$




\subsection{Static Analysis of the Reconfigurable Frame}

In the process of transforming the robot's locomotion from the wheeled mode to the tracked mode, the V-shaped link ABF rotates driven by a torque $T$ (see Fig. 5, which causes the reconfigurable frame to expand and stretch out and subsequently exerts force on the deformable track belt, through the extension wheels I and J leading to the configuration change of the tracked wheel.

Assuming that the deformation of deformable track belt is elastic with elastic deformation coefficient $k$, according to Hooke's law, referring to Fig. 5 the tensile force of deformable track belt can be expressed as

$$
F_{0}=k\left(L_{T}-L_{0}\right)=k \Delta_{L}=k \varepsilon L_{0}
$$

The tensile force $F_{0}$ is transmitted through the extension wheels and exerted on the reconfigurable frame at points I and J. Referring to Fig. 5 the forces exerted on points I and J can be obtained as

$$
\boldsymbol{F}_{I}=\frac{F_{0}}{\sin \gamma_{1}} \frac{\boldsymbol{p}_{I}}{\left\|\boldsymbol{p}_{I}\right\|} \text { and } \boldsymbol{F}_{J}=\frac{F_{0}}{\sin \gamma_{2}} \frac{\boldsymbol{p}_{J}}{\left\|\boldsymbol{p}_{J}\right\|}
$$

where $\boldsymbol{p}_{I}$ and $\boldsymbol{p}_{J}$ are obtained in Eqs. (4) and 5, and it has $\boldsymbol{p}_{I} /\left\|\boldsymbol{p}_{I}\right\|=\left(\cos \theta_{I}, \sin \theta_{I}\right)$ and $\boldsymbol{p}_{J} /\left\|\boldsymbol{p}_{J}\right\|=$ $\left(\cos \theta_{J}, \sin \theta_{J}\right)$.

Moreover, assuming that $\boldsymbol{F}_{2}=F_{2} \hat{\boldsymbol{l}}_{2}$ is the force exerted on link CD by link BC and $\boldsymbol{F}_{6}=F_{6} \hat{\boldsymbol{l}}_{6}$ is the force applied on link GH by link FG, with $\hat{\boldsymbol{l}}_{2}=\left(\cos \theta_{2}, \sin \theta_{2}\right)$ being unit direction vector of link BC and $\hat{\boldsymbol{l}_{6}}=\left(\cos \theta_{6}, \sin \theta_{6}\right)$ being unit direction vector of link FG; referring to Fig. 5 , there exist $\boldsymbol{l}_{D I} \times \boldsymbol{F}_{I}+\boldsymbol{l}_{D C} \times \boldsymbol{F}_{2}=0$ and $\boldsymbol{l}_{H J} \times \boldsymbol{F}_{J}+\boldsymbol{l}_{H G} \times \boldsymbol{F}_{6}=0$ which lead to the results that

$$
\boldsymbol{F}_{2}=\frac{F_{0}\left(l_{3}+l\right) \sin \left(\theta_{I}-\theta_{3}\right)}{l_{3} \sin \left(\theta_{2}-\theta_{3}\right) \sin \gamma_{1}} \hat{\boldsymbol{l}}_{2}
$$

and

$$
\boldsymbol{F}_{6}=\frac{F_{0}\left(l_{7}+l\right) \sin \left(\theta_{J}-\theta_{7}\right)}{l_{7} \sin \left(\theta_{6}-\theta_{7}\right) \sin \gamma_{2}} \hat{\boldsymbol{l}}_{6}
$$

Hence, referring to Fig. 5. the relation between the actuating torque $T$ and the tensile force $F_{0}$ can be expressed as

$$
\boldsymbol{T}=\boldsymbol{l}_{1} \times \boldsymbol{F}_{2}+\boldsymbol{l}_{5} \times \boldsymbol{F}_{6}
$$

that is

$$
T=\frac{F_{0} l_{1}\left(l_{3}+l\right) \sin \left(\theta_{I}-\theta_{3}\right)}{l_{3} \sin \left(\theta_{2}-\theta_{3}\right) \sin \gamma_{1}} \sin \left(\theta_{2}-\theta_{1}\right)+\frac{F_{0} l_{5}\left(l_{7}+l\right) \sin \left(\theta_{J}-\theta_{7}\right)}{l_{7} \sin \left(\theta_{6}-\theta_{7}\right) \sin \gamma_{2}} \sin \left(\theta_{6}-\theta_{5}\right)
$$

where the joint angles can be obtained from the kinematic analysis in Section 3.1 .

\section{Numerical Simulation and Parametric Study}

\subsection{Numerical Simulation and Position Synchronization}

In order to validate the working principle of the reconfigurable frame, numerical simulation is carried out in this section with the structure parameters listed in Table 1 . where $R, r, \alpha, \beta, w, p_{1}, p_{5}$ are the specified design variables and the rest of the parameters are calculated using Eqs. (9) and (10).

Table 1: Structure parameters of the reconfigurable frame in initial design

\begin{tabular}{cccccccccc}
\hline \multirow{2}{*}{ Design variable } & $R(\mathrm{~mm})$ & $r(\mathrm{~mm})$ & $w(\mathrm{~mm})$ & $p_{1}$ & $p_{5}$ & $\alpha\left(^{\circ}\right)$ & $\beta\left(^{\circ}\right)$ & & \\
\cline { 2 - 8 } & 150 & 25 & 404 & 0.5 & 0.5 & 108 & 20 & & \\
\hline $\begin{array}{c}\text { Structure parameter } \\
(\mathrm{mm})\end{array}$ & $l$ & $l_{1}$ & $l_{2}$ & $l_{3}$ & $l_{4}$ & $l_{5}$ & $l_{6}$ & $l_{7}$ & $l_{8}$ \\
\cline { 2 - 9 } & 30.0 & 106.3 & 106.3 & 126.6 & 133.0 & 106.3 & 106.3 & 126.6 & 133.0 \\
\hline
\end{tabular}




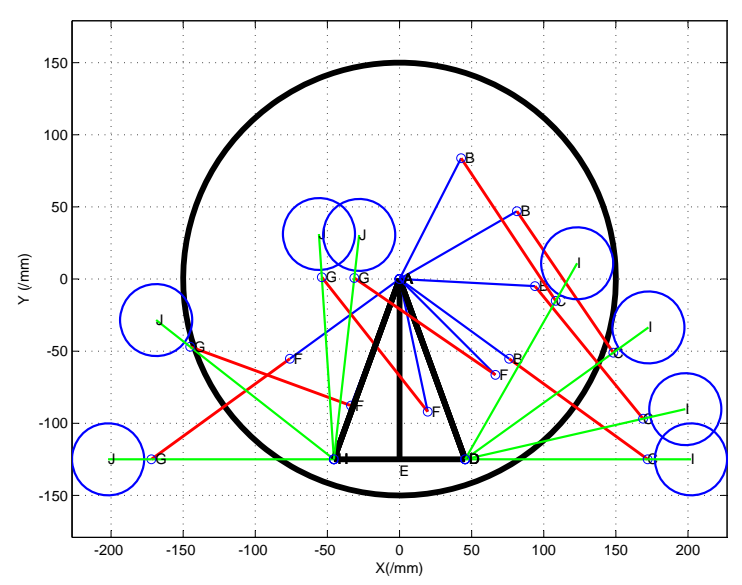

(a) Motion trajectories of extension wheels I and J

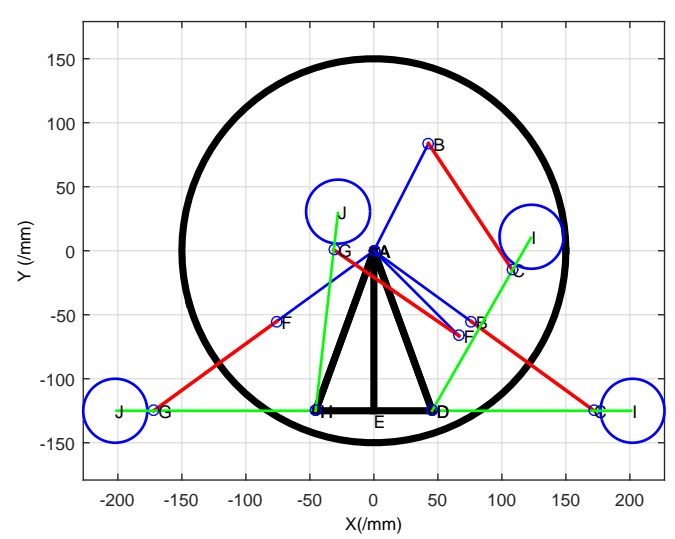

(b) Extreme cofingurations

Figure 6: Simulation of the transformation process

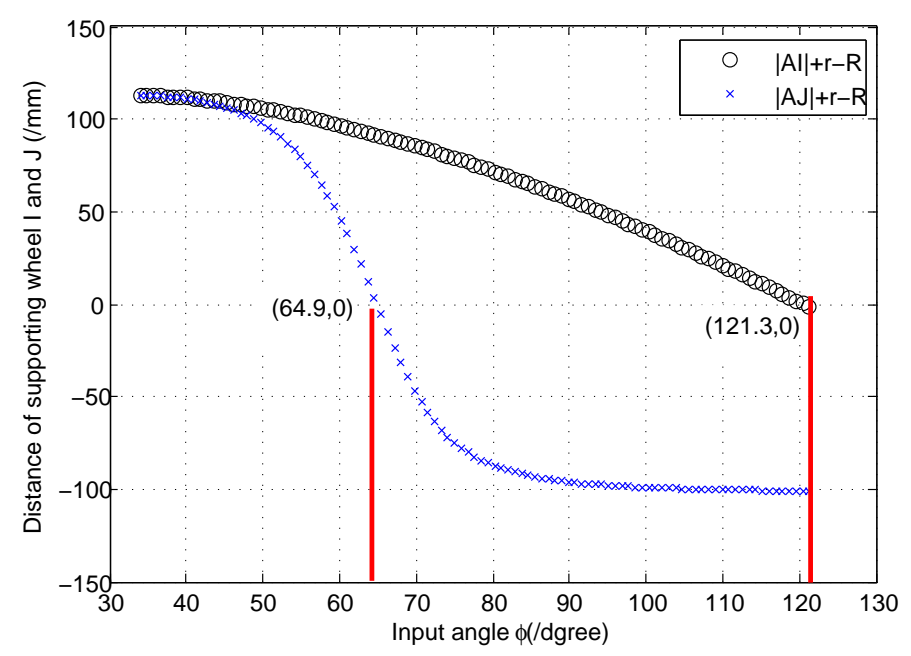

Figure 7: Locations of extension wheels I and J

170

By substituting the structure parameters into kinematic analysis Eqs. (1) throughout 7] in Section 3.1. and by assigning the input/driving angle $\phi$ with the relation that $\phi=\pi / 2-\beta+\theta_{1}$, trajectories of the extension wheels I and J from the wheeled mode to the tracked mode are illustrated in Fig. 6 .

Figure 6a shows the general configurations and Fig. 6b shows the extreme working configurations. In addition, locations of the extension wheels I and I relative to the track wheel are illustrated in Fig. 7. It can be seen from Fig. 7 that: before the driving angle $\phi$ reaches $64.9^{\circ}$, the robot works in tracked mode; when the input angle $\phi=64.9^{\circ}$, wheel $\mathrm{J}$ is contracted into the internal space of the track wheel (shown in blue ' $x$ ' dotted curve in Fig. 7] and at $\phi=121.3^{\circ}$ both of the two extension wheels are embedded in the track wheel leading to the wheeled mode. The two extreme geometric configurations of the transformational frame, namely wheeled mode and tracked mode are shown in Fig. 6b

Further, by substituting the structure parameters into Eqs. [11) throughout (14) in Section 4.1, relation between the driving angle $\phi$ and the change of length of the deformable track can be characterized as illustrated in Fig. 8.

It can be found from Fig. 8 that when the reconfigurable frame stays in the fully expanded state with $\phi=\theta_{1}=34^{\circ}$, the length change induced by the extension wheels I and $\mathrm{J}$ reaches the maximum and the total extension length of the deformable track belt is $\Delta_{L}=299.8 \mathrm{~mm}$. When the input angle increases in the interval $34^{\circ}<\phi<64.9^{\circ}$, as the wheel $\mathrm{J}$ contracts faster than the wheel I, the extension length induced 


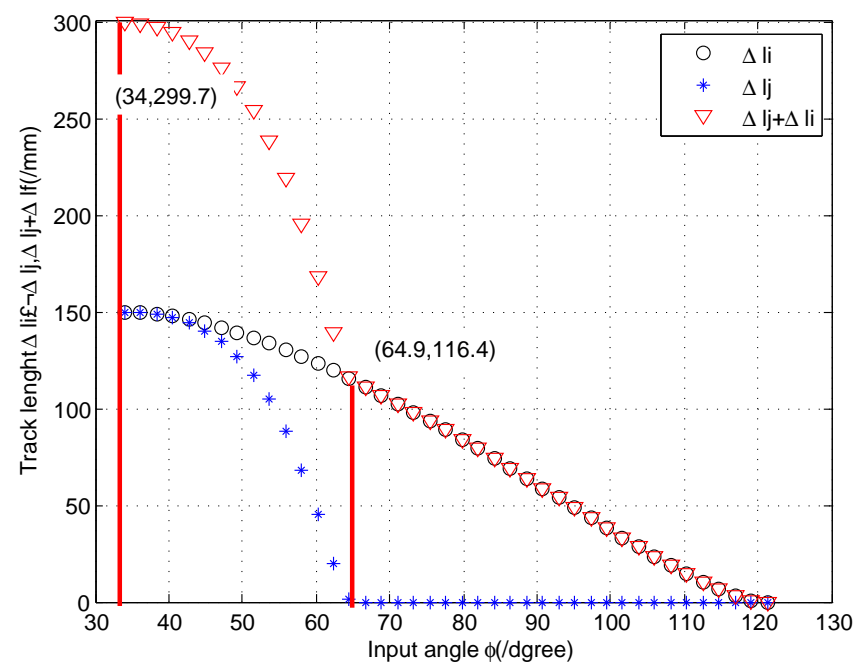

Figure 8: Change of extension length of the track belt

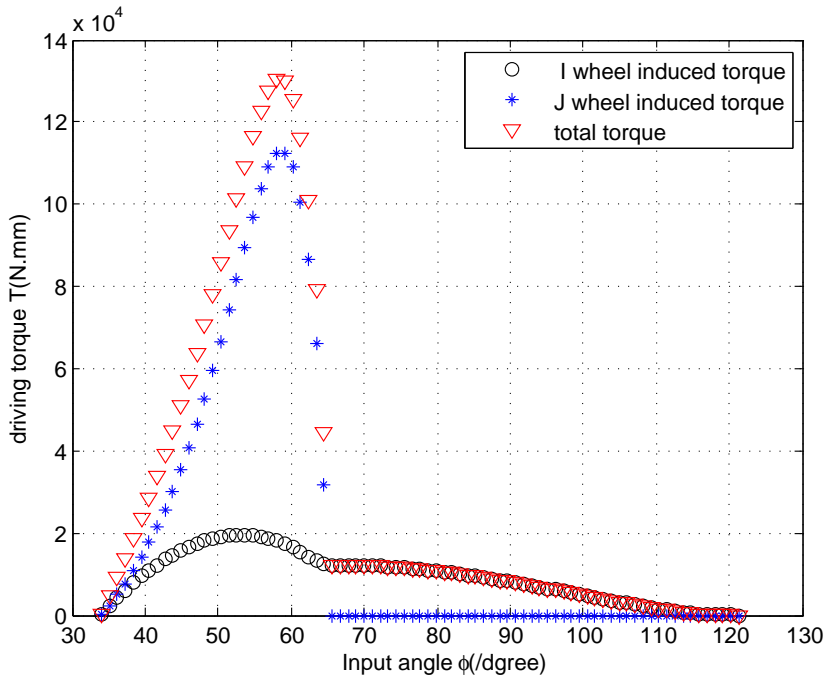

Figure 9: Torque variation with respect to input angle

by wheel J (shown in blue '*' dotted curve) decreases sharply. When the input angle $\phi=64.9^{\circ}$, wheel $\mathrm{J}$ contracts in the internal space of the track wheel. When the driving angle $\phi$ is greater than $64.9^{\circ}$ but less than $121.3^{\circ}$, wheel $\mathrm{J}$ does not alter the extension length of the deformable track belt, the extension length change induced by wheel I (shown in red ' $\nabla$ ' dotted curve) decreases gently. When the input angle $\phi$ reaches $121.3^{\circ}$, both the extension wheels I and J contract inside of the track wheel, and length of the track belt remains constant afterwards.

In addition, by substituting the structure parameters into the static analysis in Section 4.2, and assuming that the stiffness coefficient $k=1.0 \mathrm{~N} \cdot \mathrm{mm}$, forces and torque applied to the transformational frame an be revealed.

Figure 9 shows the variation of driving torque $W$. When the robot keeps in fully expanded configuration with $\phi=34^{\circ}$, the driving torques induced by wheel I (shown in black 'o' dotted curve) and wheel J (indicated in blue '*' dotted curve) are zero; this implies that the motor does not exert any torque on the $\mathrm{V}$-shaped link in the fully expanded state, which helps reduce energy consumption and protect the motor whilst the robot overcomes an obstacle. When the driving angle $\phi$ reaches $53.6^{\circ}$, the torque induced by wheel I reaches a maximum value of $1.96 \times 10^{4} \mathrm{~N} \cdot \mathrm{mm}$. When the input angle $\phi$ reaches $59.1^{\circ}$, the torque 
induced by wheel J reaches a maximum value of $11.2 \times 10^{4} \mathrm{~N} \cdot \mathrm{mm}$, and the total driving torque $W$ (in red ' $\nabla$ ' dotted curve) has a maximum value of $12.98 \times 10^{4} \mathrm{~N} \cdot \mathrm{mm}$, which is the nominal value for the driving motor.

From the numerical simulation, it can be found that during the expanding process, the extension wheels I and J lack motion synchronization such that in the fully contracted state positions of the wheels I and J are asymmetric in geometry as shown in Fig. 6a which leads to the imbalance of mass distribution and thus dynamic properties. From Fig. 9 it can be found that, the driving torque induced by the wheel I changes gently, but the driving torque induced by the wheel J contains a sharp peak at $\phi=59.1^{\circ}$ and overwhelms the driving torque induced by the wheel I. This raises the requirement for additional torque and complex control of the actuators.

Since the robot mainly works in either the fully contracted configuration as wheeled mode or in the fully stretched configuration as tracked mode, in order to improved dynamic properties for both of these two configurations and to reduce control complexity, structure parameters of the Watt II six-bar linkage is need to be modified so as to locate the wheels I and $\mathrm{J}$ in the symmetric positions in the fully contracted state as illustrated in Fig. 10 .

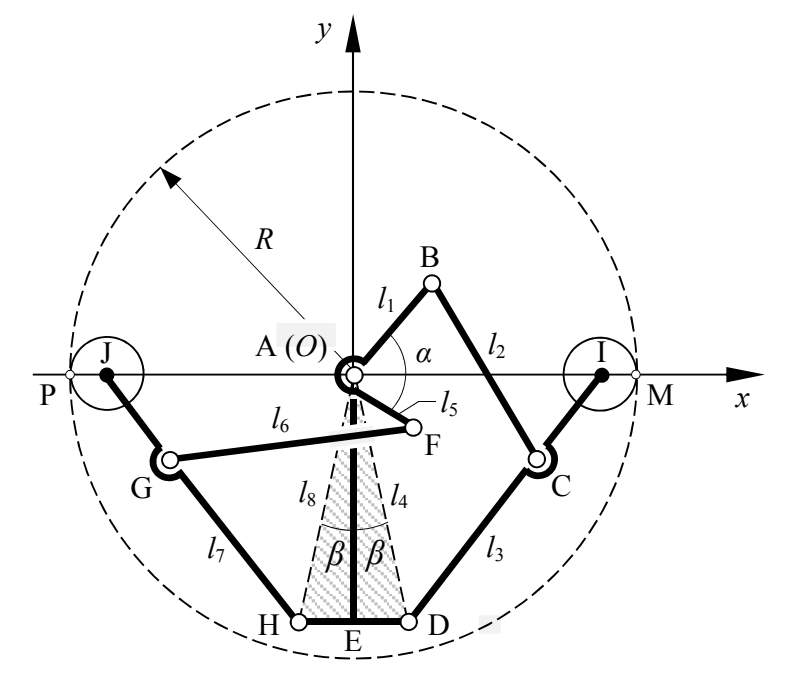

Figure 10: Desired contracted configuration of the two extension wheels

Remaining design variables of the robot the same as those in Table 1 except for $p_{5}$, we aim to achieve the above goal by modifying $p_{5}$, which is associated with the lengths of links $l_{5}$ and $l_{6}$. Referring to Fig. 10 let wheel I be tangential to the track wheel at point $\mathrm{M}$ and lies on the $x$-axis, the goal is to adjust the link lengths of links $l_{5}$ and $l_{6}$ such that wheel $\mathrm{J}$ is tangential to track wheel at point $\mathrm{P}$ and lies on the $x$ axis, symmetrically with respect the the $y$-axis. It can be seen that in this configuration, point $\mathrm{G}$ can be determined through two routes, i.e., route $\mathrm{A} \rightarrow \mathrm{F} \rightarrow \mathrm{G}$ and route $\mathrm{A} \rightarrow \mathrm{H} \rightarrow \mathrm{G}$, and they can be expressed as

$$
\left[\begin{array}{cc}
\cos \theta_{5} & \cos \theta_{6} \\
\sin \theta_{5} & \sin \theta_{6}
\end{array}\right]\left[\begin{array}{l}
l_{5} \\
l_{6}
\end{array}\right]=\left[\begin{array}{cc}
\cos \theta_{7} & \cos \theta_{8} \\
\sin \theta_{7} & \sin \theta_{8}
\end{array}\right]\left[\begin{array}{l}
l_{7} \\
l_{8}
\end{array}\right]
$$

As the wheels $\mathrm{J}$ and I are symmetric, as shown in Fig. 10. there must exist $\theta_{7}=\pi-\theta_{3}, \theta_{8}=3 \pi / 2-\beta$, $\theta_{5}=2 \pi-\alpha+\theta_{1}, l_{6}+l_{5}=l_{1}+l_{2}, l_{7}=l_{3}$ and $l_{8}=l_{4}$. By substituting these relations into Eq. 21 and eliminating $\theta_{6}$, it yields

$$
\left\{\begin{array}{l}
l_{5}=\frac{L_{L}^{2}-X_{H}^{2}-Y_{H}^{2}}{2\left(L_{L}-X_{H} \cos \left(2 \pi-\alpha+\theta_{1}\right)-Y_{H} \cos \left(2 \pi-\alpha+\theta_{1}\right)\right)} \\
l_{6}=l_{1}+l_{2}-l_{5}
\end{array}\right.
$$

where, $L_{L}=l_{1}+l_{2}, X_{H}=l_{3} \cos \left(2 \pi-\alpha+\theta_{1}\right)+l_{4} \cos (3 \pi / 2-\beta), Y_{H}=l_{3} \sin \left(2 \pi-\alpha+\theta_{1}\right)+l_{4} \cos (3 \pi / 2-\beta)$.

Using Eq. 22, we can calculate the optimized link lengths $l_{5}$ and $l_{6}$, which can ensure the symmetry of the extension wheels J and I in the fully contracted state. 
Corresponding to the original structure parameters in Table 1 a new set of structure parameters corresponding to the position synchronization are obtained as listed in Table2

Table 2: Structure parameters of the reconfigurable frame with motion synchronization

\begin{tabular}{|c|c|c|c|c|c|c|c|c|c|}
\hline \multirow{2}{*}{ Design variable } & $R(m m)$ & $r(m m)$ & $w(m m)$ & $p_{1}$ & $p_{5}$ & $\alpha\left(^{\circ}\right)$ & $\beta\left(^{\circ}\right)$ & & \\
\hline & 150 & 25 & 404 & 0.5 & 0.31 & 108 & 20 & & \\
\hline \multirow{2}{*}{$\begin{array}{l}\text { Structure parameter } \\
(\mathrm{mm})\end{array}$} & $l$ & $l_{1}$ & $l_{2}$ & $l_{3}$ & $l_{4}$ & $l_{5}$ & $l_{6}$ & $l_{7}$ & $l_{8}$ \\
\hline & 30.0 & 106.3 & 106.3 & 126.6 & 133.0 & 65.4 & 147.2 & 126.6 & 133.0 \\
\hline
\end{tabular}

Using these semi-optimized structure parameters and adopting the programs used in Section 5.1 the improved trajectories of the extension wheels I and J can be computed and illustrated in Fig. 11a We find that trajectories of the wheels I and J are more or less synchronized. In the fully contracted state, the two wheels I and J are symmetric, which helps keep dynamic balance in the wheeled mode. Meanwhile, using these modified parameters, the driving torque of the robot is illustrated in Fig. $11 \mathrm{~b}$ Comparing with the results before motion synchronization (see Fig. 9, it can be found that, the torque induced by wheel J vary gently and the peak torque reduces from $11.2 \times 10^{4} \mathrm{~N} \cdot \mathrm{mm}$ to $3.3 \times 10^{4} \mathrm{~N} \cdot \mathrm{mm}$, and the total torque decreased from $13.0 \times 10^{4} \mathrm{~N} \cdot \mathrm{mm}$ to $5.7 \times 10^{4} \mathrm{~N} \cdot \mathrm{mm}$. This reduces the requirement of complementary torque from the actuator.

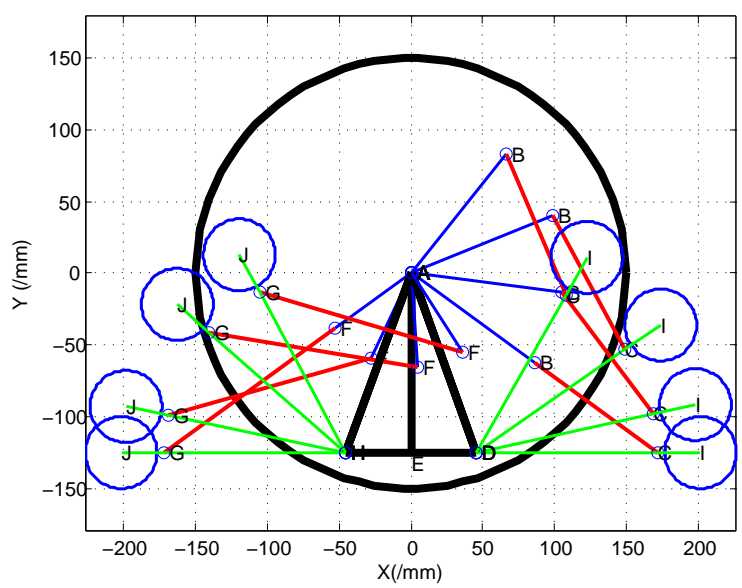

(a) Improved motion trajectories

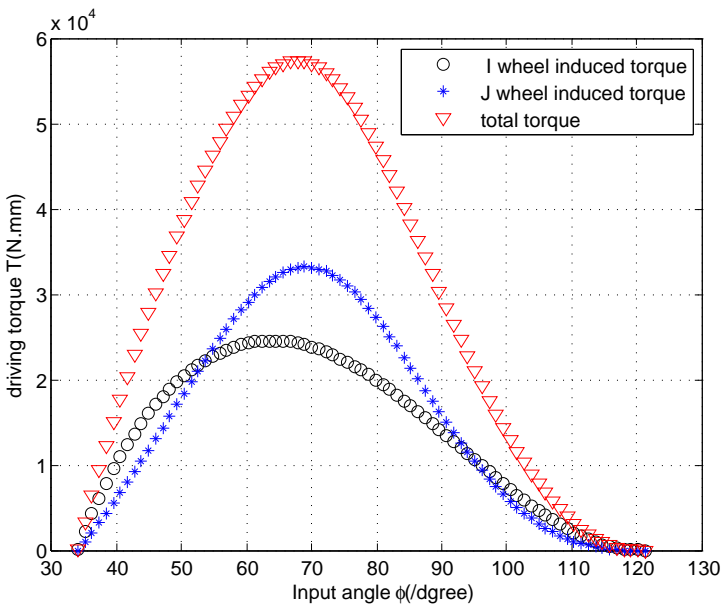

(b) Optimized driving torque

Figure 11: Optimized trajectories and driving torque

\subsection{Driving-torque Associated Parametric Study and Optimization}

As discussed in section 5.1, by changing design variable $p_{5}$, approximate motion synchronization of wheels I and $\mathrm{J}$ is achieved which helps reduce the driving torque of the motor and provide a better dynamic balance. In order to further optimize of the structure parameters to reducing the driving torque, we remain $p_{5}, R, r$ and $w$ the same as listed in Table 2 and adjust the values of $p_{1}, \alpha$ and $\beta$ based on the parametric study method. Using this method, the relationship between design variables $\left(p_{1}, \alpha, \beta\right)$ and the driving torque $T$ will be investigated through graphic based parametric study; leading to an optimized set of structure parameters for the reconfigurable mechanism.

Firstly, we choose $\left(p_{1}, \alpha, \beta\right)=\left(0.5,108^{\circ}, 20^{\circ}\right)$ as initial value which has been obtained in Section 5.1; keeping the values of $\alpha$ and $\beta$ unchanged, and we vary the value of $p_{1}$ so as to reveal the relationship between $p_{1}$ and the driving torque $T$. Plotting the relationship in graph as shown in Fig. 12a it indicates 


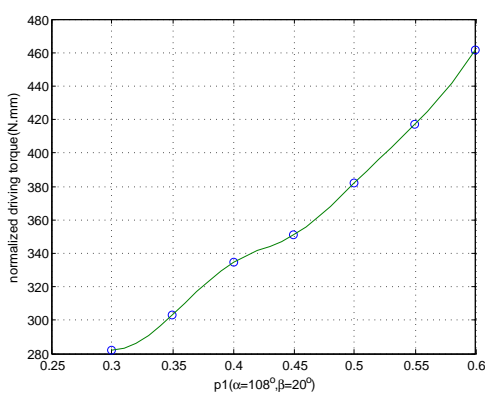

(a) $p_{1}$ vs driving torque

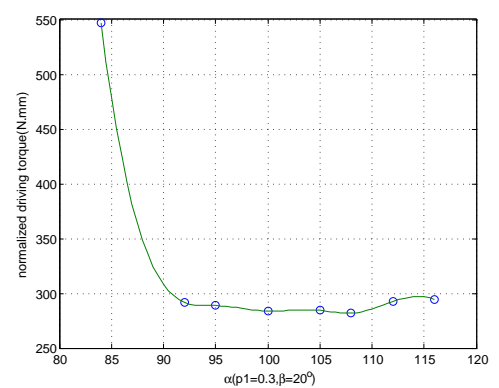

(b) $\alpha$ vs driving torque

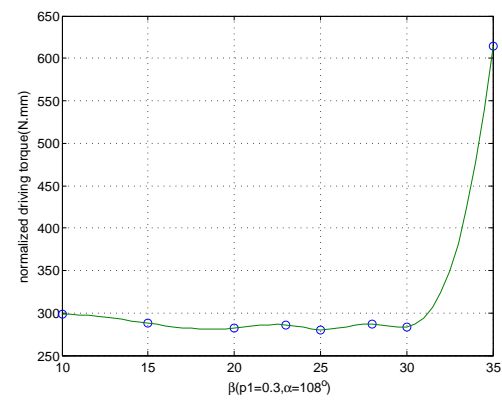

(c) $\beta$ vs driving torque

Figure 12: Driving-torque associated parametric study

that the driving torque $T$ increase with $p_{1}$, the minimum torque is $281.80 \mathrm{~N} \cdot \mathrm{mm}$ when $p_{1}=0.3$; hence the best value to choose is $p_{1}=0.3$.

Subsequently, let $p_{1}=0.3$ and $\beta=20^{\circ}$, we investigate the influence of the variation of $\alpha$ on the driving torque. Fig. $12 \mathrm{~b}$ shows that the angle $\alpha$ has a non-linear effect on the driving torque; the minimum torque of $281.80 \mathrm{~N} \cdot \mathrm{mm}$ occurs when $\alpha=108^{\circ}$.

Further, let $p_{1}=0.3$ and $\alpha=108^{\circ}$, we study the relationship between the angle $\beta$ and the driving torque. As shown in Fig. 12c we found that the effect of value $\beta$ on the driving torque is also non-linear; the minimum driving torque of $280.25 \mathrm{~N} \cdot \mathrm{mm}$ arises whilst $\beta=25^{\circ}$.

Therefore, through the above driving torque associated parametric study, a set of better values for the design variables $p_{1}, \alpha$ and $\beta$ are obtained as $\left(p_{1}, \alpha, \beta\right)=\left(0.3,108^{\circ}, 25^{\circ}\right)$; and using these values together with the other design variables listed in Table 2 a new set of optimized structure parameters can be calculated as listed in Table 3 . It should be pointed out that the value of design variable $p_{5}$ varies with the changes of values of lengths of link $l_{5}$ and $l_{6}$.

Table 3: Optimized structure parameters of the reconfigurable frame

\begin{tabular}{|c|c|c|c|c|c|c|c|c|c|}
\hline \multirow{2}{*}{ Design variable } & $R(m m)$ & $r(m m)$ & $w(m m)$ & $p_{1}$ & $p_{5}$ & $\alpha\left(^{\circ}\right)$ & $\beta\left(^{\circ}\right)$ & & \\
\hline & 150 & 25 & 404 & 0.3 & 0.24 & 108 & 25 & & \\
\hline \multirow{2}{*}{$\begin{array}{l}\text { Structure parameter } \\
(\mathrm{mm})\end{array}$} & $l$ & $l_{1}$ & $l_{2}$ & $l_{3}$ & $l_{4}$ & $l_{5}$ & $l_{6}$ & $l_{7}$ & $l_{8}$ \\
\hline & 30.0 & 63.8 & 148.9 & 113.8 & 137.9 & 50.9 & 161.8 & 113.8 & 137.9 \\
\hline
\end{tabular}

Substituting the structure parameters in Table 3 into the kinematic and static equations in Sections 3 and 4, Fig. 13a shows the fully expended and folded configurations and Fig. 13b shows the variation of driving torque with respect to input angle $\phi$; it can be found that the torque induced by the extension wheel J changes gently and the peak torque reduces from $3.3 \times 10^{4} \mathrm{~N} \cdot \mathrm{mm}$ to $2.5 \times 10^{4} \mathrm{~N} \cdot \mathrm{mm}$, and the total torque decreases from $5.7 \times 10^{4} \mathrm{~N} \cdot \mathrm{mm}$ to $4.2 \times 10^{4} \mathrm{~N} \cdot \mathrm{mm}$.

\section{Prototype Development and Field Tests}

Based on the theoretical analysis presented above, using the structure parameters obtained in Table 3 with a some essential adjustments required from mechanical component design, a physical prototype of the proposed RHMBot was developed as shown in Fig. 14. This robot consists of a mobile robot and a remote operating terminal. The mobile robot, as shown in Fig. 14 a, includes a mechanical module, a driving module, a braking module, a communication module and an embedded micro-controller; the remote operating terminal, as shown in Fig. 14 p, includes a communication module, a vision module and a remote controller. The robot can be transformed into three locomotion modes as aforementioned, i.e. the wheeled mode in Fig. 14 a, the tracked mode in Fig. $14 \mathrm{c}$ and the roll-over mode in Fig. 14 d. 


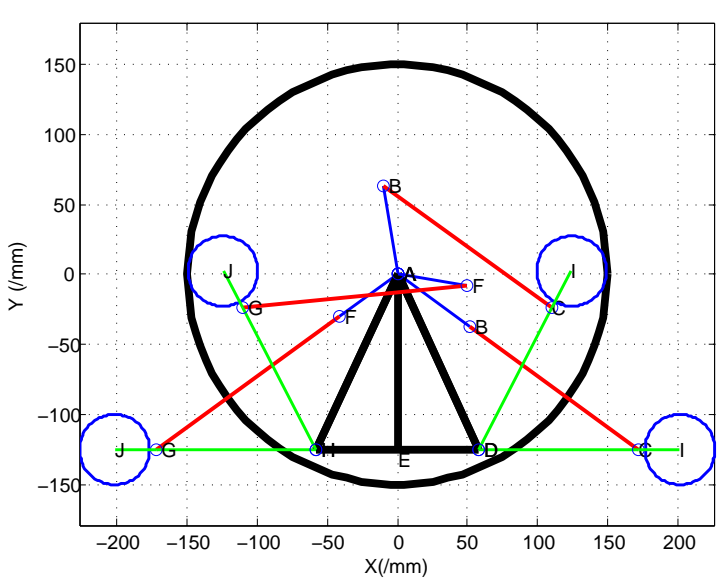

(a) Improved motion trajectories

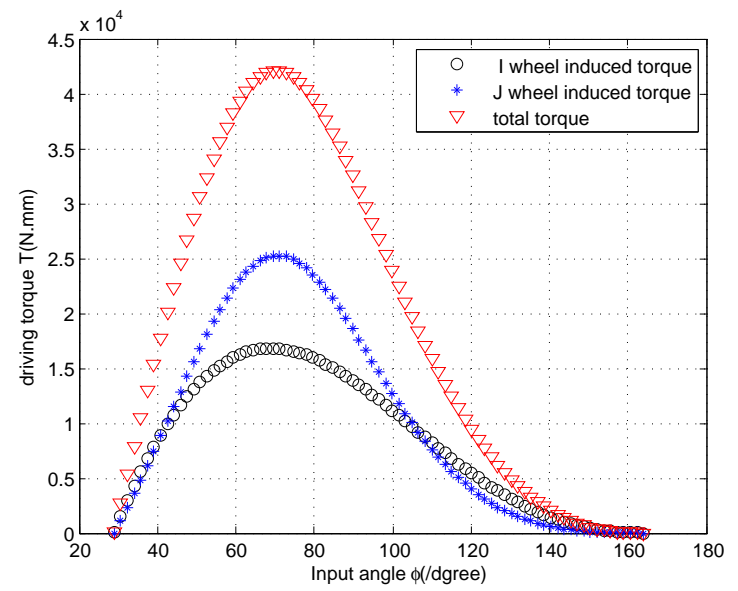

(b) Optimized driving torque

Figure 13: Optimized trajectories and driving torque

For the deformable track belt, the elastic layer is made of latex through impregnation process, it has high elongation rate, good elasticity and low cost; the constraint layer is made of polyamide through casting process, the wavy shape makes it possible to elongate with the elastic layer, and maintain a constant total length of the track; the transmission layer is divided into pieces by belt with tooth profile consistent with the track, and the elastic layer, constraint layer and transmission layer are riveted and bonded together. During the transformation process, shape of the deformable track belt changes according to the configuration of the reconfigurable frame, and the total length of the deformable track belt alters with respect to the corresponding driving angle $\phi$. We find that the maximum length is $1395 \mathrm{~mm}$, which is greater than the value of $1242 \mathrm{~mm}$ indicated in Section 5.1 before the optimization. This indicates that the optimized design parameters make the robot possess greater geometric dimension in the expanded state, which helps promote obstacle-overcoming ability of the robot. Figures. $15 \mathrm{~B}$ and $15 \mathrm{~b}$ show the reconfigurable frame and the tracked wheel of the prototype, and Figs. $15 \mathrm{c}$ and $15 \mathrm{~d}$ illustrate the deformable track belt in two configurations. The reconfigurable mechanism was actuated and tested and the video screen shorts indicated in Figs. $16 \mathrm{a}$ to $16 \mathrm{~d}$ show that the robot can not only fulfil the locomotion reconfiguration from the wheeled mode to the tracked mode, but also satisfy the geometric constraints and the driving torque requirement.

Based on the prototype, a series of field tests were carried out so as to check and verify the performance of the proposed mobile robot. A video for the tests is available in the supplementary material for this paper. During the mobile experiments, an operator controlled the remote operating terminal and sent out control command, which was received by the communication module on the mobile robot and subsequently translated into control signal to drive and control the actions of the robot, including locomotion mode transformation, start-stop, steering and so on.

When the robot runs on even grounds, grass surfaces or hard terrains, it usually works in the wheeled mode to achieve higher efficiency and velocity. When the robot encounters large obstacles or complicated terrains, it can transform itself from the wheeled mode into the tracked mode such that the circular tracked wheels are transformed into the triangular tracks. And the robot is consequently transformed into a tracked robot which helps improve obstacle-overcoming ability and adapt itself to work on the gravel, sand and other complicated terrains that are not suitable for a wheeled robot.

In the case that the robot comes across extremely large obstacles that are hard to conquer in the wheeled mode or the tracked mode, it then turns into the roll-over mode. In this mode, the robot keeps itself in a triangular track configuration and rotates the triangular track like a wheel to climb and roll-over the obstacle. In the roll-over mode, the robot can continuously climb and roll-over vertical steps of 120 $\mathrm{mm}$ high, i.e. $80 \%$ of the robot's axle height, as demonstrated in Figs. $17 \mathrm{a}$ to $17 \mathrm{~d}$. It can be seen from Figs. 17. that the maximum height that the robot can actually overcome is much higher than the height of step. 


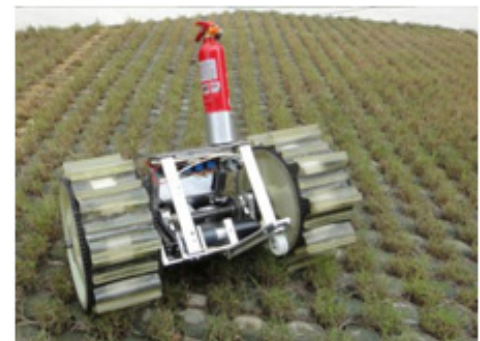

(a)

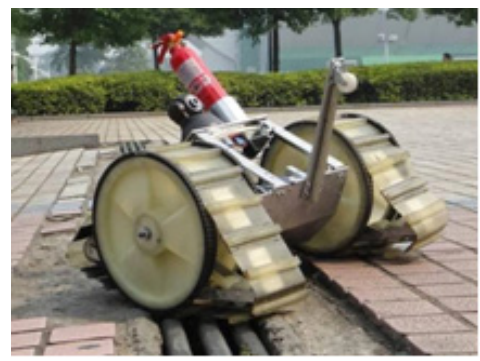

(c)

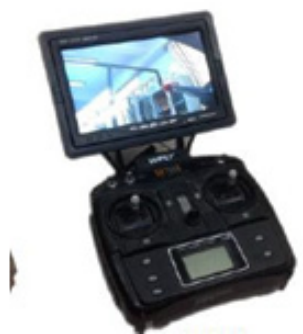

(b)

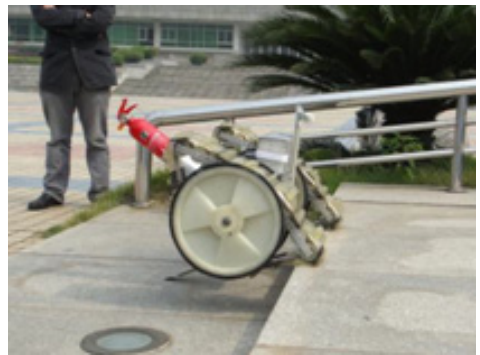

(d)

Figure 14: Physical prototype of the RHMBot

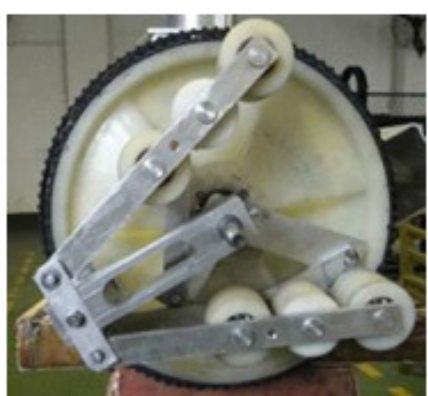

(a)

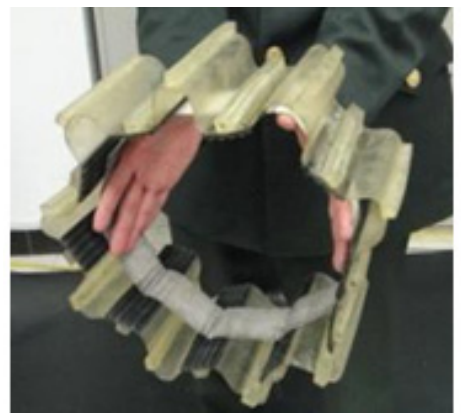

(c)

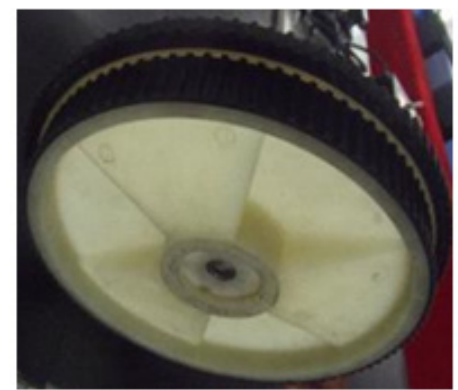

(b)

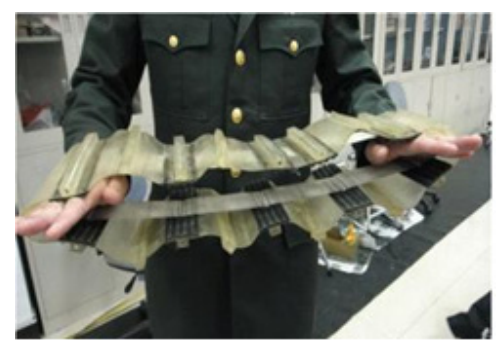

(d)

Figure 15: The transformational frame and the deformable track belt 


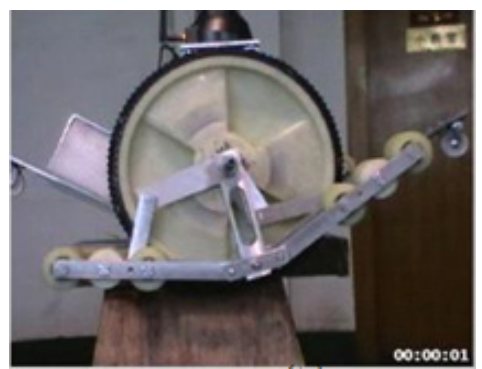

(a)

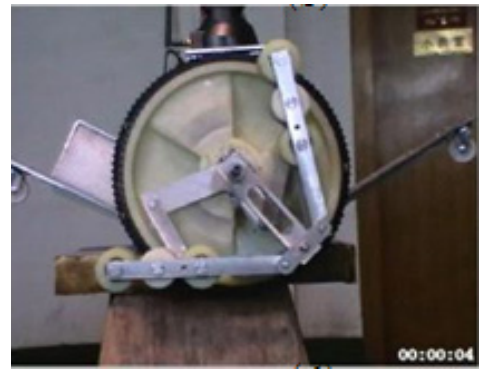

(c)

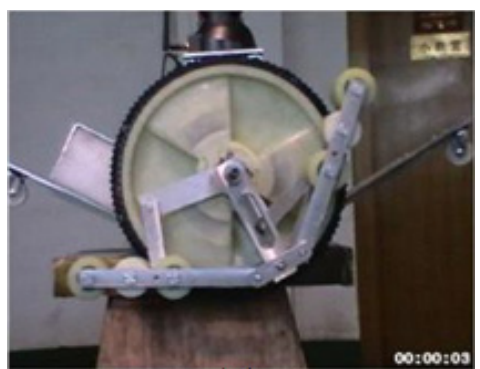

(b)

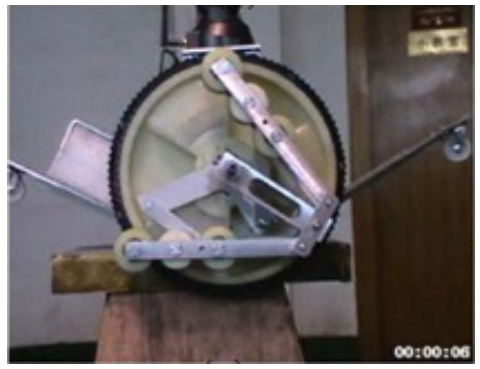

(d)

Figure 16: Transformation of the reconfigurable frame

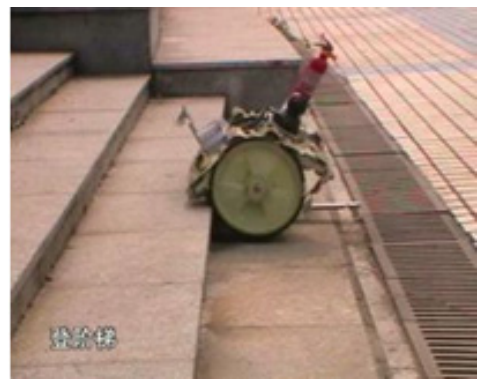

(a)

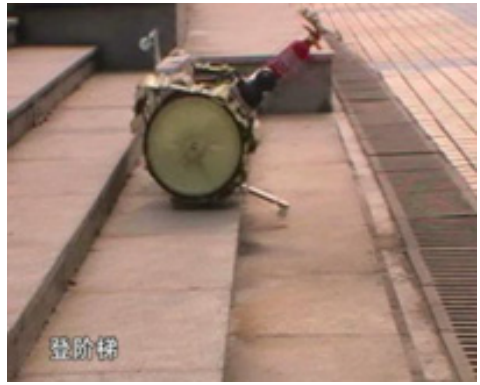

(c)

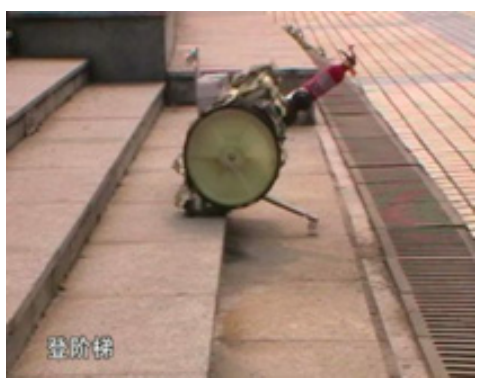

(b)

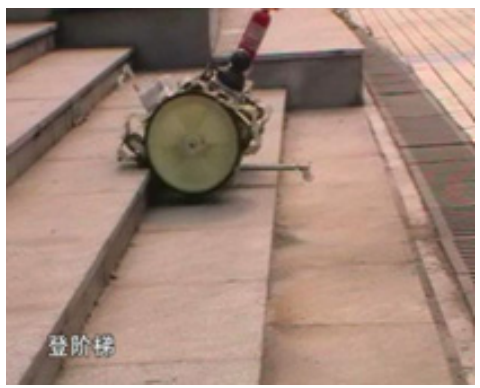

(d)

Figure 17: Robot climbs steps in the roll-over mode 


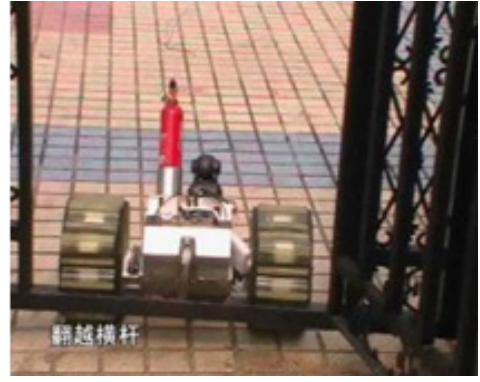

(a)

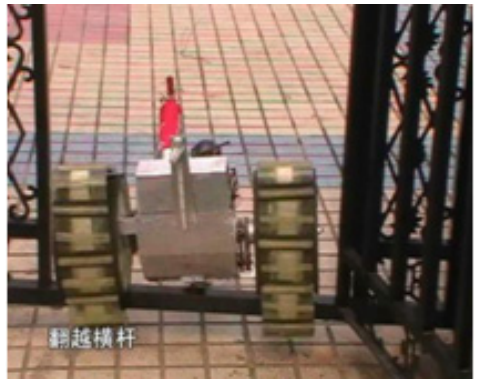

(c)

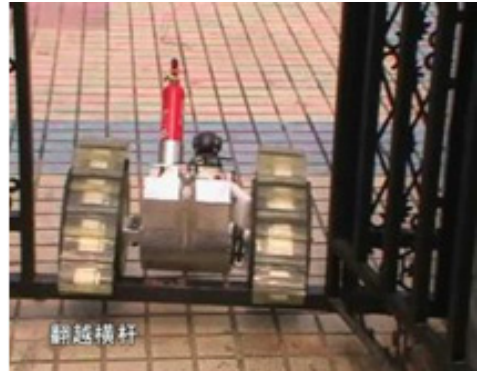

(b)

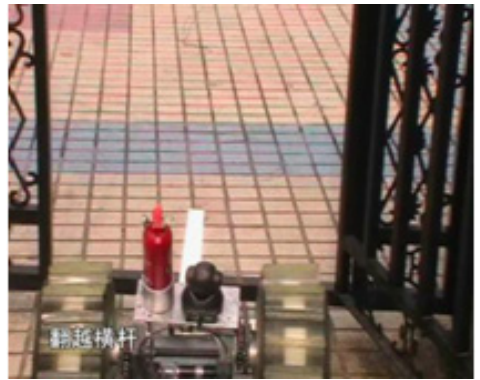

(d)

Figure 18: Robot overcomes a railing in the roll-over mode

Further, Figs. 18 a to $18 \mathrm{~d}$ show that the robot can climb and overcome a $140 \mathrm{~mm}$ high railing. When the robot faces a railing that is hard to conquer in the wheeled mode and the tracked mode (see Fig. 18a), the robot will transform itself into the tracked mode and rotates the triangular track as a whole to climb over the railing (see Fig. 18b). Supported by the rotation torque provided by the tail frame, the triangular track rotates continuously (see Fig. 18 f) until the robot rolls over the railing successfully as shown in Fig. $18 \mathrm{~d}$.

The tests conducted through the physical prototype demonstrated that the actual obstacle-overcoming ability is determined not only by the geometric factors but also by the robot posture, friction condition, driving torque and other factors during the obstacle overcoming process. The tests accomplished here not only verify the mathematical model, optimization and simulation in this paper, but also shows the fact that the robot developed based on the proposed design principles has distinctive obstacle-overcoming capability.

\section{Conclusions}

This paper for the first time proposed and developed a novel reconfigurable hybrid wheel-track mobile robot (RHMBot). The robot can implement three different locomotion modes: wheeled mode, tracked mode and roll-over mode. Reconfigurability of the proposed robot is achieved by the creative design through the combination of a deformable track belt with a reconfigurable frame constructed based on a Watt II six-bar linkage.

Mechanical design of the proposed mobile robot was presented and kinematic and static analysis of the reconfigurable frame associated with the deformable track belt were formulated. Through numerical simulation the transformation function, force/torque properties and geometric constraints as well as performance of the deformable track belt were characterized and illustrated.

Based on position synchronization of the two extension wheels and driving-torque associated parametric study, structure parameters of the proposed mobile robot were optimized; leading to the semioptimized parameters which resulted in the development of a physical prototype of the RHMBot. Field tests were subsequently carried out to prove the design concept, feasibility, manoeuvrability and multiple locomotions of the proposed mobile robot. The test results indicate that the RHMBot can reconfigure itself to adapt to different terrains. It can work in wheeled locomotion model under even terrain and 
transform into triangular track model upon encountering small obstacles, and reconfigure into climbing and roll-over mode in the case that larger obstacles are encountered.

The novel robot presented in this paper thus has provided new insights into the design of hybrid mobile robot with multiple locomotion modes, which has potential applications in the fields of homeland security, surveillance, reconnaissance in dangerous situations, and space exploration.

\section{Acknowledgement}

The authors gratefully acknowledge the support from the Natural Science Foundation of China (NSFC) under grant number 51475465 .

[12] A. Halme, I. Leppanen, S. Salmi, Development of workpartner-robot: design of actuating and motion control system, in: 2nd International Conference on Climbing and Walking Robots (CLAWAR 99), 1999, pp. 657-666.

[13] X. G. Duan, Q. Huang, J. K. Li, Design and motion analysis of miniature wheel-track-legged mobile robot, Chinese Journal of Mechanical Engineering 41 (8) (2005) 108-114.

[14] X. G. Duan, Q. Huang, J. T. Li, Design and implementation of a small ground mobile robot with multilocomotion models, China Mechanical Engineering 18 (1) (2007) 8-12. 
[15] H. T. Feng, Y. Ou, X. Y. Gao, Dynamics modeling and analysis of miniature ground-moving robot based on special pose, Journal of Nanjing University of Science and Technology 30 (4) (2006) 486-

[16] J. S. Dai, J. Rees Jones, Mobility in metamorphic mechanisms of foldable/erectable kinds, Transactions of the ASEM, Journal of Mechanical Design 121 (3) (1999) 375-382.

[17] J. S. Dai, M. Zoppi, X. W. Kong (Eds.), Advances in reconfigurable mechanisms and robots I, SpringerVerlag, London, 2012.

[18] B. M. Yamauchi, Packbot: a versatile platform for military robotics, in: Proceedings of the SPIE, Vol. 5422, 2004, pp. 228-237, doi:10.1117/12.538328.

[19] Z. Li, S. Ma, B. Li, M. Wang, Y. Wang, Design and basic experiments of a transformable wheel-track robot with self-adaptive mobile mechanism, in: The 2010 IEEE/RSJ International Conference on Intelligent Robots and Systems, Taipei, 2010, pp. 1334-1339.

[20] S. K. Lim, D. I. Park, Y. K. Kwak, B.-S. Kim, S.-W. Jeon, Variable geometry single-tracked mechanism for a rescue robot, in: IEEE International Workshop on Safety, Security and Rescue Robotics, 2005, pp. 111-115.

[21] Y.-G. Kim, J.-H. Kwak, J. Kim, J. An, K.-D. Lee, Adaptive driving mode control of mobile platform with wheel-track hybrid type for rough terrain in the civil environment, in: 2010 International Conference on Control Automation and Systems (ICCAS),, 2010, pp. 86-90.

[22] F. X. Govers, Elbit tracks \& wheels viper, Robot Magazine 2009 (2009) 24-25.

[23] Y. Spector, D. Raz, A. Novoplanski, G. Rinberg, Adaptable traction system of a vehicle, patent, US 7334850 B2 (Feb. 2008).

[24] Z. Luo, J. Shang, L. Xi, L. Ren, J. Yang, Z. Wang, A reconfigurable hybrid wheel-track robot, patent, CN102849132 B (Nov. 2014).

[25] M. M. Plecnik, J. M. McCarthy, Numerical synthesis of six-bar linkages for mechanical computation, Transactions of the ASME, Journal of Mechanisms and Robotics 6 (3) (2014) 031012.

[26] A. G. Erdman, G. N. Sandor, S. Kota, Mechanism Design: Analysis and Synthesis, Vol. 1, Prentice Hall, Englewood Cliffs, New Jersey, 2001.

[27] K. J. Waldron, G. L. Kinzel, S. K. Agrawal, Kinematics, Dynamics, and Design of Machinery, 3rd Edition, Wiley \& Sons, Ltd., Chichester, West Sussex, 2016.

[28] J. M. McCarthy, G. S. Soh, Geometric Design of Linkages, Springer, New York, NY, 2010. 